\title{
Miranda
}

Revue pluridisciplinaire du monde anglophone /

Multidisciplinary peer-reviewed journal on the English-

speaking world

19 | 2019

Rethinking Laughter in Contemporary Anglophone Theatre

\section{« Naissance des comiques gays et lesbiens américains : le rire queer comme performance esthético-politique »}

Xavier Lemoine

\section{OpenEdition}

1 Journals

Édition électronique

URL : http://journals.openedition.org/miranda/20106

DOI : 10.4000/miranda.20106

ISSN : 2108-6559

Éditeur

Université Toulouse - Jean Jaurès

Édition imprimée

Date de publication : 7 octobre 2019

\section{Référence électronique}

Xavier Lemoine, « «Naissance des comiques gays et lesbiens américains : le rire queer comme performance esthético-politique » », Miranda [En ligne], 19 | 2019, mis en ligne le 09 octobre 2019, consulté le 16 février 2021. URL : http://journals.openedition.org/miranda/20106 ; DOI : https:// doi.org/10.4000/miranda.20106

Ce document a été généré automatiquement le 16 février 2021.

\section{cc)}

Miranda is licensed under a Creative Commons Attribution-NonCommercial-NoDerivatives 4.0 International License. 


\title{
« Naissance des comiques gays et lesbiens américains : le rire queer comme performance esthético- politique »
}

\author{
Xavier Lemoine
}

1 La place centrale du rire dans le corpus des spectacles queer laisse à penser que le rire est une caractéristique constitutive de la représentation queer. En effet, depuis l'avènement du camp ${ }^{1}$ dans les années 1960 chez Jack Smith à la satire sociale de Penny Arcade (Longing Lasts Longer, joué en octobre 2014 au Public Theatre à New York), à l'autodérision des années SIDA, le rire pourfend les conventions attachées à la sexualité, au genre et à la racialisation. Qu'il soit provoqué par le trait d'esprit, l'humour, la provocation, la revendication, l'auto-défense, ou plus généralement par la manifestation de l'étrangeté au sein d'un système normatif, le rire est un ingrédient récurrent des spectacles queer. Si le rire de la parodie postmoderne révèle les mécanismes de pouvoir normatif et suggère une possibilité d'action (Hutcheon 6; Butler 1990) pour se défaire, se déprendre de l'emprise des conventions, alors il n'est pas étonnant de le retrouver sans cesse au cœur des spectacles queer. Mais qu'en est-il de cette forme de spectacle entièrement dédiée au rire : le spectacle comique ou standup comedy. Comme le rappelle le comique/performer latino John Leguizamo, il existe une certaine confusion sur les termes « (...) some say it's performance art, others say it's stand-up, or others say theater piece and others say comedic one-man show.» (Russell 236) Il propose néanmoins une définition :

Stand up, you usually find a persona for yourself, and then from there you tell a lot of JPMs (jokes per minute) and they're all stream-of-consciousness, and they all don't have to relate to each other. You tell little bites, little riffs, they can tell stories, but it's not as theatrical". (Russell 236-7)

2 Pour Leguizamo, le stand-up est un genre distinct qui s'inspire de comiques tels Richard Pryor ou Lenny Bruce (références incontestées du genre). C'est un art de la condensation et du raffinement : « I want to do a stand-up show, because I feel that (...) 
it challenges me in terms of creating jokes and just making them quicker and sharper. It's like a haiku. » (Russell 237) Le stand-up serait donc une fabrique du rire, épuré de sa pesanteur théâtrale, et donc l'endroit idéal, trop évident peut-être, pour explorer le sens du rire sur scène. Existe-t-il alors un rire queer et dans quelle mesure la pratique du comique éclaire cette notion critique et théorique?

3 Afin de mieux saisir les liens qui se tissent entre rire et queer, il semble pertinent d'établir une perspective historique sur l'émergence et le développement du « stand-up gay et lesbien ». Plusieurs ouvrages (Stone, Karvosky, Limon) soulignent la naissance du comique gay visible dans les années 1970, notablement avec la comique lesbienne militante Robin Tyler (Karvosky 12). Puis dans les années 1980, des soirées gays et lesbiennes sont accueillies dans les salles de spectacles des grandes villes (particulièrement à San Francisco). Enfin, dans les années 1990 grâce à la demande de spectacles due à la croissance des chaînes câblées, les comiques homosexuels font leur entrée sur les plateaux de télévision. L'évolution de ce genre semble ouvrir des perspectives stimulantes entre les histoires personnelles gays, lesbiennes, trans et les enjeux sociaux de la représentation queer. Cette tendance se fait à la faveur d'un boom comique général stimulé par le profit et une actualité politique riche en événements (Stone xiii; xix). La critique du Village Voice Laurie Stone dans son ouvrage sur les humoristes Laughing in the Dark éclaire sans ambages la nature commerciale du standup :

The growth of comedy, on the other hand, is driven not by a grassroots initiative but by money. The organizers are club owners and producers scouting product. Comedy is showbiz, which means buck. (Stone xiii)

Le stand-up serait un art moins noble aux yeux d'une certaine critique intellectuelle car trop proche de l'industrie du divertissement. Paradoxalement, la recherche artistique ou culturelle en ferait alors un genre mineur en dépit de son omniprésence. Par-là même, cependant, il rejoint une dimension définitionnelle du queer, sa dimension minoritaire. Le rire pourrait être un point d'entrée dans la réflexion queer, balayant des définitions trop étroites de la subjectivation, et rebattant les cartes catégorielles de la scène. En d'autres termes, dans quelle mesure le rire queer propose-t-il une redéfinition non seulement d'un genre mais encore des processus d'élaboration des sujets hybrides contemporains?

On peut identifier quelques déclinaisons du rire queer: le rire politique, le rire esthétique et le rire subversif. À travers un stand-up politique se déploie dans de nouveaux espaces un combat contre le rire homophobe afin de laisser advenir le sujet gay, lesbien, bi et trans* (LGBT+) via les narrations de coming out, les contre-discours et les «contrepublics $»^{2}$. Au mieux, ce rire serait un mode opératoire du processus de « désidentification » tel que le définit Muñoz:

Disidentification is meant to be descriptive of the survival strategies the minority subject practices in order to negotiate a phobic majoritarian public sphere that continuously elides or punishes the existence of subjects who do not conform to the phantasm of normative citizenship (Muñoz 1999: 4)

6 Le rire n'est cependant pas seulement un acte de résistance et d'opposition mais une tentative d'invention de nouveaux espaces et de nouvelles perceptions. En effet, le rire camp traverse les scènes et s'impose comme une force de subversion des genres masculins/féminins, de la sexualité, de la race afin de faire exister le sujet queer hybride. Il s'agit d'explorer le camp dans sa nature politique, comme l'avait proposé Moe Meyer dans l'introduction de son ouvrage collectif The Politics and Poetics of Camp 
(Meyer 7), mais aussi dans sa mécanique classique détaillée dans un numéro de Critique de 1988 consacré au rire. Tous les contributeurs s'accordent à caractériser le rire par son éclat. La montée du rire serait irrépressible, irréconciliable, et éphémère. Ainsi, le rire n'est pas une essence (Nancy) et, par-là, l'emploi de rire gay/queer ne défend pas une catégorie essentialiste mais, au contraire, traque la remise en circulation des identifications. Ces déclinaisons, (rire militant, rire subversif) ne sont donc qu'une configuration passagère, en déclin, pour proposer une trace possible du rire dans l'histoire de la performance queer.

\section{Stand-up LGBTQ (queer) : le rire militant}

7 Le spectacle comique LGBT a-t-il la moindre fonction politique ? Crée-t-il une alliance entre rire et homosexualité afin d'alimenter le mouvement queer? En d'autres termes, le stand-up queer participe-t-il à une mise en échec du système hétéronormatif, genré, racialisée de la société ? Plus encore est-il la chute d'un ordre social, ou à tout le moins un point de rupture? En proposant une généalogie du stand-up LGBT américain, l'on peut tenter d'apporter des éléments de réponse à ces questions générales grâce à des exemples précis.

8 Logiquement, l'on peut considérer que les spectacles comiques gays et lesbiens ${ }^{3}$ s'inscrivent dans le développement de la visibilité et des droits politiques gay et queer des années 1980 et 1990. Ils ont accompagné l'explosion dans les années 1980 de petits clubs à New York City qui ont donné jour à une multiplication de spectacles par des comiques gays/queer (Stone xiii) sur les deux côtes américaines. À l'intérieur de l'espace catégoriel du stand-up les comiques queer viennent brouiller les frontières $\mathrm{du}$ genre. Le stand-up est une catégorie friable, enfant de la tradition du Vaudeville - ces spectacles hybrides mêlant comiques, cirques, danse, strip-tease etc. Allen \& Wilmeth 390-1) - qui est de nouveau modifié profondément par l'inclusion d'une subjectivité queer.

\section{Espaces pour rire gay}

9 La question d'espace, de lieu des possibles, est un aspect primordial de l'émergence du stand-up LGBT. À San Francisco le Valencia Rose Cabaret, serait le premier théâtre comique gay et lesbien du pays (Karvoski 13, 27). La comique lesbienne latina Marga Gomez se souvient de ses débuts et confirme l'importance de ce lieu :

Founded in 1982, The Valencia Rose Cabaret was an ambitious project that housed the world's first gay comedy club, a queer activist meeting center, a vegetarian eatery and more into a two-story Spanish style building with 3D camel sculptures jutting out of its walls.

[...] As a new comic and a Latina, I could barely get any stage time as it was. One day I noticed a flyer on a telephone pole about a "Gay Comedy Night" at The Valencia Rose.

I showed up that Monday in a red vintage dress. The room was full. I told my first gay joke which involved an old lady telling me, "It's good to see dykes wearing dresses again." They laughed and I got something I hadn't experienced from any audience till then; love. (Marga Gomez)

10 Dès la naissance des espaces comiques gay, la dimension culturelle, communautaire, sociale et politique est frappante. Le sentiment d'appartenir à une communauté chez 
Gomez est presque naïf mais n'en demeure pas moins profond lorsqu'elle ressent « l'amour » du public au Valencia. Cette impression est caractéristique du renversement de la polarité alors dominante entre la haine ressentie dans les circuits comiques hétérosexuels et ce nouvel espace d'amour. Une autre comique latina queer, Monica Palacios, corrobore qu'elle se sentait en sécurité au Valencia (Karvoski 43) et presque tous les comiques de cette époque évoquent cette idée d'un changement profond des perceptions entre un espace gay et non gay.

11 À New York plusieurs scènes s'ouvrent, hébergeant toute sorte de spectacles tant sur le plan thématique que formel. " The Duplex », ouvert dans les années 1950, au cœur de ce qui deviendra le quartier gay historique de la ville se consacre à la comédie gay mais pas uniquement (comme le site internet le rappelle fièrement, Woody Allen y a fait ses débuts). D'autres espaces accueillent les comiques queer mais aussi des spectacles dont la nature est mixte entre comédie, performance et pièces de théâtre : "Dixon Place ", "WOW Café », " Joe's Pub au Public Theater », « PS 122 » etc. Cette effervescence sur les planches permettra, grâce à la porosité entre scène et écran de télévision, de voir passer dix ans plus tard des comiques gays et lesbiens à la télévision.

12 Les premières apparitions à la télévision seront dans les niches procurées par la croissance des chaînes privées payantes dont une chaîne entièrement dédiée aux comiques (Comedy Central). Le besoin de remplir des grilles de 24 heures entraîne une recherche intensive de comiques et un passage de la scène à l'écran quasi automatique. Ainsi la comique butch hyper-active Lea DeLaria est l'hôtesse de la première émission télévisée consacrée aux comiques queer Out There, le 11 octobre 1993, avant une seconde édition en 1994 (Karvoski 14). Même si ce changement de medium n'est pas sans conséquence, la circulation du comique queer entre le petit écran, le cinéma, la grande salle, et l'espace alternatif fait partie des enjeux politiques de représentations. Les comiques dans les années 1980 et 1990 ont acquis une visibilité en rupture avec les décennies précédentes et ont ouvert la possibilité d'un sujet LGBT multiple au-delà d'identités médicalisées et marginalisées.

13 Cette évolution implique non seulement les artistes mais également le rapport au public, alimentant la réflexion queer et l'invention d'un contrepublic défendue par l'universitaire Michael Warner :Après avoir redéfini la notion de public, en insistant sur la dimension relationnelle et l'adresse publique impersonnelle, Warner identifie un contrepublic queer :

Their members are understood to be not merely a subset of the public, but constituted through a conflictual relation to the dominant public. They are structured by different dispositions or protocols from those that obtain elsewhere in the culture, making different assumptions about what can be said or what goes without saying. In the sense of the term that I am here advocating, such publics are counterpublics, and in a stronger sense than simply comprising subalterns with a reform program. A counterpublic maintains at some level, conscious or not, an awareness of its subordinate status. The cultural horizon against which it marks itself off is not just a general or wider public but a dominant one. And the conflict extends not just to ideas or policy questions, but to the speech genres and modes of address that constitute the public, or to the hierarchy media. (Warner 423-4)

14 Warner explicite bien l'émergence d'une résistance d'un contrepublic contre les dispositifs hétéronormatifs et homophobes. Les comiques LGBT et leur contrepublic illustrent ce mécanisme clairement en convoquant l'existence du public queer par ce nouveau genre et cette nouvelle interpellation. Lors des émissions Out There, les plans 
sur les divers couples homosexuels sont appuyés et témoignent d'un changement qui se passe aussi bien sur scène que dans la salle. Le rire queer se construit à travers le public queer. C'est-à-dire que ce n'est pas seulement un comique queer qui semble naître à cette période-là mais également un public queer. L'un n'existe pas vraiment sans l'autre : comme le suggère Limon, à la suite de Freud, ${ }^{4}$ dans son ouvrage, Stand-up Comedy in Theory, Or, Abjection in America, la blague n'existe que par le rire de son public. Pour lui, l'éclat de rire est bien ce qui distingue le stand-up comique du spectacle contenant simplement de l'humour (qui n'implique par l'éclat de rire) : pas de rire, pas de blague, pas de comique, pas de stand-up, et on pourrait ajouter pas de queer. Limon exprime cette co-dépendance et cette co-création repérée également par les théoriciens de la performance (Dwight Conquergood puis E. Patrick Johnson) :

Audiences turn their [comedians'] jokes into jokes, as the comedian had not quite

thought or expressed a joke until the audience thinks or expresses it. (Limon 13)

Selon Warner, le contrepublic n'est pas défini comme une simple identité ou communauté. C'est la relation, le contexte et la circulation qui fondent son existence. Parallèlement, dans notre perspective, ce n'est pas le public mais son rire qui est déterminant. Par extension, on pourrait conclure que le rire queer serait la clé de voûte du stand-up queer, voire même de toute la communauté assemblée (ce contrepublic queer). Cette possibilité du spectacle comique serait un signe de la transformation de la société dans sa capacité à pouvoir accueillir un nouveau rire. Mais qu'est-ce qui a donc déclenché ce rire à l'époque?

\section{Rire contre l'homophobie?}

16 Alors que le rire devenait hétérosexuel grâce à l'exclusion du point de vue minoritaire homosexuel imbriqué dans l'exclusion raciste, misogyne (etc.), le rire queer briserait les chaînes de l'homophobie régnante non pas tant en inversant les rôles (se moquer des hétérosexuels, même si cela n'est pas exclu) mais en transformant l'oppression, voire l'insulte, l'injure, en potentiel de prise de liberté, en champ du possible de la blague. ${ }^{5}$

Le Valencia, selon les témoignages de nombreux comiques, devient un espace de liberté où l'omerta sur l'homosexualité, outre les moqueries, est désagrégée. Le bon mot de son directeur artistique évoque bien ce sentiment du point de vue du contrepublic:

Donald Montwill, who became its artistic director in the second year, once said that queer audiences had been waiting for this place "where they could enjoy comedy and not be the butt of the joke, pun intended." (Gomez)

18 En effet, comme le souligne Laurie Stone, nombreux sont les comiques qui ont utilisé la misogynie et l'homophobie dans le passé (Bob Hope, Stone 5) mais aussi plus récemment (Stone 46 et le témoignage de Paul Jacek, Karvoski 189-90). Dans l'émission de télévision comique des années 1990 produite par la Fox, In Living Color, les comiques noirs Damon Wayans et David Allen Grier jouent les rôles d'Antoine Merriweather et Blaine Edwards (ensemble de 12 sketchs programmés d'avril 1990 à février 1993) deux homosexuels stéréotypés à l'excès, entre autres parce qu'ils sont efféminés et maniérés. Dans le contexte grand public de la télévision, la moquerie insultante plutôt que la complicité subversive empêche une réception intersectionnelle de ce rire. L'universitaire E. Patrick Johnson s'interroge sur la répétition d'une homophobie dans le contexte de la masculinité hétérosexuelle noire, plus particulièrement à travers l'humour homophobe d'un Eddy Murphy ou de Wayans et Grier. Il souligne aussi la possibilité de 
queeriser ces représentations (Johnson 61-75) par la réappropriation. C'est justement ce que la circulation de la télévision à la scène, du grand public au contrepublic permet à la troupe afro-américaine gay "Pomo Afro Homos ». Le chorégraphe et danseur Djola Bernard Branner, le comédien Brian Freeman, et le chanteur, danseur et comédien Eric Gupton dans leur spectacle de 1991 Fierce Love joué à Josie's Cabaret à San Francisco (Roman 165-166) subvertissent l'homophobie des sketchs de Wayans et Grier. Dans le numéro d'ouverture de ce spectacle, un militant du fictionnel ACT-BLACK zappe ${ }^{6}$ les critiques de cinéma Antoine et Blaine, joués par les deux autres membres de la troupe. Ainsi le spectacle retourne-t-il le rire contre la production d'un comique hétéronormatif ancré dans l'angoisse d'une masculinité noire menacée ontologiquement par le féminin et l'homosexualité. Cette parodie souligne à la fois les limites du groupe militant contre le SIDA, ACT UP, insuffisamment conscient des enjeux raciaux dans ses engagements et la circulation d'une image dégradante ou extrêmement limitée de ce que serait un homosexuel noir. Plus encore, la réappropriation des Pomo Afro Homos œuvre à désamorcer le stéréotype d'un "negro faggot » dénoncé par l'influent artiste queer afro-américain Marlon Riggs. Dans son article « Black Macho Revisited: Reflections of a Snap! Queen ", Riggs produit un «SNAP » discursif contre les caricatures homophobes de l'homosexuel noir déployé par le rap, la télévision et le cinéma noir. Tout le monde n'était cependant pas nécessairement choqué, considérant le sketch comme une parodie outrancière à dessein s'accordant avec la logique invoquée par Wayans pour défendre sa liberté d'humoriste. Wayans et Grier, toujours prêts à réaffirmer leur hétérosexualité, incarnent néanmoins une version de l'homophobie persistante au début des années 1990. Plus exactement, au-delà de leurs intentions, ils signalent que la moquerie de l'homosexuel efféminé fait toujours recette. La différence tient à ce qu'un contre-rire est proposé grâce à la troupe « Pomo Afro Homos ».

Les comiques en permettant de rire avec et non contre l'homosexualité redéfinissent les limites du rire. Si le rire naît de la transgression, alors à une époque où l'homophobie est la norme les blagues sur les gays et les lesbiennes seraient mécaniquement transgressives. Et quelque part les comiques considérés homophobes jouent avec cet interdit. Le public dominant se rassure de ne pas faire partie du groupe minoritaire dont le comique se moque, plus encore, il est constitué par le rejet instinctif et physiologique du rire. Néanmoins, comme le suggère l'essai de Mikkel Borch-Jacobsen le rire n'est pas seulement disjonctif mais peut se placer davantage du côté de l'empathie :

Riant de ce passant qui glisse sur une peau de banane (ou, cela revient exactement au même, riant de l'absurde destinée d'(Edipe), je ne ris pas d'être ce que l'autre n'est pas - supérieur, souverain, auto-suffisant, etc. Au contraire, je ris en participant joyeusement à sa chute, en glissant avec lui dans cette soudaine et souveraine nullité qui est la sienne. (Borch-Jacobsen, 32)

Sans doute, le rire homophobe (mais aussi misogyne, raciste) reste sur le premier versant: je ris «d'être ce que l'autre n'est pas» et je suis dans l'illusion phantasmatique d'être "supérieur ", de croire que l'hétérosexualité, la masculinité, la race échappent à la construction phantasmatique de l'identification (Butler 1993: 93-119). L'avènement des comiques LGBT, permet de réaliser la seconde proposition d'une chute commune, d'une compréhension que les incongruités rencontrées par les homosexuels participent pleinement à l'humanité et à ses limites. Le potentiel de queerisation est bien sûr politique puisqu'il touche aux rapports de pouvoir mais contribue aussi aux ressorts profonds du rire. La logique du rire queer participe à un 
élargissement de l'humanité, en incluant plusieurs groupes (voire en groupes) jusque-là exclus, et en redéfinissant les contours de l'humain par ses failles. En d'autres termes, les mécanismes d'identification sont mis au jour ce qui permet de produire ce que Butler décrit ainsi : " a dynamic map of power in which identities are constituted and/ or erased, deployed and/or paralyzed » (Butler 1993 : 117)

Dans les années 1970, le rire gay était peu répandu car l'homophobie résistait encore à son effondrement structurel. 1982 est donc un moment politique pour les comiques grâce à la création d'un espace de liberté, si petit soit-il, dans les faits quelques salles, quelques soirées, afin de pouvoir s'esclaffer queer. La filiation entre le comique et le politique est littérale sur la côte Ouest si l'on considère que l'un des fondateurs du Valencia Rose, Hank Wilson, était associé à Harvey Milk, le célèbre élu municipal de San Francisco assassiné le 27 novembre 1978 (voir le film Harvey Milk, 2008). Ensemble, ils ont lutté contre une proposition de loi de l'État de Californie destinée à interdire aux homosexuels d'être enseignants (Marche 79). Le rire queer est donc bien politique dans sa lutte contre l'oppression homophobe à travers ses représentations culturelles (Wayans) ou sociales (limitations des droits). La dimension politique et épistémologique n'est pas seulement sociale ou systémique (anti-homophobe), elle est aussi en conjonction avec des thématiques personnelles qui permettent de mettre en place des stratégies de résistance grâce au stand-up LGBT.

\section{Le rire performatif?}

\section{Les blagues de coming out}

Il est délicat de faire une cartographie exhaustive des thématiques des comiques queer, dans la mesure où le stand-up est un art hautement contextuel, le rire est intimement lié à un moment et à un public et il y a donc autant de blagues que d'événements (politiques, culturels, médiatiques etc.), que d'individus ${ }^{7}$ et que de réceptions. Souvent les comiques tirent leurs gags de la presse comme c'était le cas avec The Joan Jett Blakk Show au «Josie's Cabaret \& Juice Joint " (Karvoski 160). Néanmoins, quelques grands thèmes sont récurrents tant sur le plan individuel que social dès les premiers spectacles des années 1980. Tout particulièrement la mise en scène comique du coming out, de l'annonce à sa famille ou à son entourage de son homosexualité, est si fréquente que ce thème semble constituer un passage particulier dans le stand-up gay et lesbien. En effet, la question du coming out est à la fois au cœur des politiques de visibilité LGBT et elle fait partie du processus d'identification de l'homosexualité. Elle est un point de tension entre le privé et le public, le caché et le manifeste propice à déclencher le rire. Si les blagues autour du coming out sont devenues un trope identifiable des spectacles gays et lesbiens, c'est sans doute qu'elles ont valeur d'acte performatif de l'identité comique LGBT, énonçant et créant à la fois le sujet queer et le genre même de ce comique. Ainsi la version la plus classique est-elle le récit de la révélation de son homosexualité aux parents, acquérant le statut d'un rituel spectaculaire qui inaugure le rire queer.

Monica Palacios transforme ce moment potentiellement tragique en comédie grâce à la diversité des réactions de sa famille :

Finally the time was right. I gathered everybody around the room and I said, 'My family - mi familia - this is my wife!' Everybody stopped talking. (...) After a moment of thick intense silence, my mom says: 'Come on, everybody, let's eat. Food is getting cold. C'mon, andale. [...] 
My Mom: ‘We know that you are...but we don't want to know again” [...]

And my precocious niece: « LEZBO » (Karvoski 46-7) profondeur du rire semble sourdre de l'exposition de la double oppression liée à l'identité lesbienne noire. La substitution de noir à gay met le doigt sur une invisibilisation double que le corps et le discours de Sykes habitent simultanément en pleine lumière. Syke donne corps à une hypothèse loufoque en jouant les dialogues entre elle et sa mère, ses silences, en incarnant sa mère, ses intonations saturées d'émotions exposant l'ignorance de la mère mais aussi de la société tout entière. La communauté noire religieuse est parodiée mais aussi la communauté LGBT blanche qui doit aussi réfléchir au sens de faire son coming out en tant qu'africaine-américaine. Le rire éclate sur l'autel du racisme et de l'homophobie ouvrant la perspective d'une désidentification intersectionnelle. La tension entre l'acceptation et le rejet du sujet par sa famille ne peut pas faire l'économie des questions raciales mais ne peut pas non plus faire l'impasse sur les autres caractéristiques de subjectivation tel qu'ici le lesbianisme. L'articulation entre ces tensions fonde la force de son sketch et la fulgurance d'une gêne profonde qu'il faut vite congédier ou embrasser grâce au rire. En interpénétrant des scripts historiques de libération du sujet, Sykes desserre l'étau normatif qui fixe les identités, elle désamorce l'emprise hétéronormative et la dilue dans l'hilarité. Cet effet irradie la comique et son (contre)public qui peuvent se désidentifier à l'expérience lesbienne noire, occupant avec Sykes une position impossible (irréelle) et malgré tout rendue réelle par la présence de la performance, au moins le temps d'une histoire drôle. Ainsi, la conscience des oppressions multiples signale la nature complexe de ce rire qui peut mener à un supplément politique. 


\section{Politique du gag gay}

27 La politique de la visibilité dépasse donc le cadre familial, elle s'inscrit dans le champ culturel tout entier. Le comique Danny Williams suggère, non sans un certain sens de la provocation, cet état de fait en abordant des sujets politiques des années 1990 :

I think our invisibility in the media is why the far right was able to demonize us in

the discussion of gay men and lesbians in the military. (Karvoski 69)

La montée de la droite ultra conservatrice, accompagnant la révolution conservatrice des années Reagan, n'est pas le fait de l'absence de comique gay - voire plutôt l'inverse. Toutefois, l'impact de la visibilité, même s'il ne va pas modifier les positions des extrémistes, est bien en cause dans le ferment culturel menant à des positions d'ouverture ou d'ostracisme. Les débats sur l'acceptation des homosexuels dans l'armée étant au cœur de l'actualité de la fin des années 1980 et au début des années 1990, montrent bien l'importance cruciale des médias dans la fabrication de cette blague. Précisément, l'actualité, par ses choix de sujet, devient prescriptive de ce qui entre dans le champ du visible et donc de ce qui détermine la politique de la visibilité. Le nombre de comiques faisant référence à ce sujet est un indicateur clair. Lynda Montgomery, par exemple, souligne son plaisir de la pratique de «show and tell » du primaire comme la source d'inspiration de son désir d'être sur scène et l'oppose brillamment à la formule produite par le consensus homophobe de Clinton « Don't Ask Don't Tell » qui l'a conduite à être une comique lesbienne :

Somehow, everything worked out as it should ; my love of show-and-tell came back into my life in the form of standup comedy. (And my discomfort with 'Don't ask, don't tell' led me to be an out lesbian comic!) (Karvoski 206)

L'homosexualité devient donc un outil politique pour ces comiques, une façon d'intervenir dans les débats publics sans tomber, toutefois, dans un positionnement naïf ou simpliste. Williams, en effet, ironise sur l'illusion des bienfaits de la visibilité dans la mesure où, bien sûr, les ultra-conservateurs ne changeraient pas d'idée même s'il y avait une meilleure visibilité des gays et lesbiennes dans les médias. Néanmoins, le rire constitue un arsenal pour se défendre des charges conservatrices - rire offensif et défensif à la fois.

Ainsi, cette montée de la droite ultra-conservatrice et religieuse se retrouve dans de nombreux sketchs (Bob Smith), révélant l'inscription contextuelle profonde des comiques et le conflit violent entre les positions queer et conservatrices alimentant les Culture Wars. Une guerre des mots, d'influence où le rire démasque les tensions extrêmes des systèmes normatifs en cours de réajustement et la constitution d'un contrepublic actif. Plusieurs comiques, Sykes comme nous venons de l'évoquer, utilisent la substitution terminologique/thématique à l'intérieur d'un canevas normatif mobilisant un rire subversif. Bob Smith dans «Under the influence» contrecarre le discours stigmatisant du chef des conservateurs au Sénat de janvier 1995 à janvier 1999, Newt Gingrich, en substituant un terme à un autre pour le décontextualiser et le recontextualiser, produisant cette distance et ce déplacement de la performance de la désidentification'. Smith puise dans les discours où Gingrich compare l'homosexualité à l'alcoolisme. Gingrich, inspiré par la pathologisation de l'homosexuel à la fin du $19^{\mathrm{e}}$ et au début $20^{\mathrm{e}}$ siècle, produit un canevas homophobe que Smith pastiche en remplaçant toutes les occurrences d'alcoolisme par gay ce qui crée une distorsion comique :

(Gay blackouts do happen. Many public figures go their entire lives being gay and not remembering it.) 
It's alarming to consider that Mr. Gingrich is capable of influencing the direction of our government's public health policies. This further the stigmatization of homosexuality could even lead to the formation of new organizations with homophobic agendas - groups such as MADG (Mothers Against Driving Gay) (Karvoski 142)

31 La version parodique de l'association Mothers Against Drunk Driving (MAAD) fait rire par la mécanique d'une substitution loufoque, mais elle est aussi clairement politique par l'allusion à la responsabilité du politicien envers la défense de la santé publique. En outre, Smith met en avant le processus de déshumanisation propagé par Gingrich en le ridiculisant avec ses comparaisons insensées. L'inhumanité grotesque se retourne contre le politicien et ses angoisses conservatrices. L'homophobie politisée est donc dénoncée par le comique grâce à cette réappropriation ludique d'une homosexualité omniprésente qui déclenche l'hilarité d'un contrepublic qui s'oppose à une clôture du sujet queer par la stigmatisation.

Globalement, la visibilité politique du comique LGBT est hybride car elle implique un coming out structurel intégré aux spectacles. C'est bien un potentiel politique spectaculaire défini par la performance qui est en jeu. L'artiste en mobilisant son homosexualité de façon performative met en abyme la caractéristique du spectacle dans sa totalité puisqu'il est construit comme une sorte de coming out: je suis lesbienne donc mon spectacle est lesbien. En effet, la comique lesbienne existe par l'énonciation même de cette identité mais cette identification dépasse l'individu pour devenir un genre comique. Ce genre comique puise sa force dans une histoire en résistance contre la fixité et reste donc un genre ouvert, fluide, déterritorialisant. Sa nature performative est essentielle tant par sa valeur d'instanciation du sujet dans le présent que par sa nécessaire réitération dans l'espace public. Ainsi, la nature performative de l'éclat de rire valide la présence qui peut servir à définir, plus généralement, le spectacle vivant (Lemoine 2016: 179). Le rire est donc une alliance politique mais aussi artistique garantissant simultanément la politique de visibilité et l'art de la présence. Le retournement du rire moqueur en rire complice serait la transformation qui qualifie aussi l'art de la scène: la production d'une mutation dans le champ de la représentation. Le charisme de Lea DeLaria ou de Sandra Bernhard illustrent le rire queer et suggèrent la nature politique de l'art de la subversion. Gilmore dans sa typologie définit la présence de DeLaria comme une sorte d'offensive permanente :

For example Lea Delaria is a lesbian Bitch comic who constantly pushes the envelope, delivering her particular 'in your face' brand of stand up to gay and straight audience alike. (Gilbert 2013 189)

33 À l'autre bout de cette typologie, Sandra Bernhard serait la version « lesbienne chic » dont l'énergie irrévérencieuse de ses spectacles (Confession of a Pretty Lady; I'm Still Here Damn It, 1998) perturbent les conventions. Ces formes de présence offrent des modèles de rire actif, affirmant la puissance plutôt que l'impuissance et donc un renversement de la domination hétéronormative des comiques.

Le rire politique est donc une première étape, presque binaire dans la mesure où il s'agit souvent d'inversion des rôles, comme le rappelle Butler « Heterosexuality does not have a monopoly on exclusionary logic » (Butler 1993 : 112) mais le rire et le queer (celui qui critique les dérives normatives des mouvements gays et lesbiens) s'accordent aussi car ils ne sont pas des catégories fermées. La réflexion du philosophe Jean-Luc Nancy aide à cerner ce caractère insaisissable du rire : 
(...) le rire n'a jamais proprement trouvé sa place dans la dialectique éroticoesthétique de la philosophie. [...] Mais le rire 'comme tel', celui qui traverse toutes ces catégories esthétiques, psychologiques ou métaphysiques sans se plier à aucune d'elles, celui qui s'empare au moins aussi de l'angoisse et de la joie, celui-là reste en marge. (Nancy 44)

Le rire comme marge, le rire comme un processus fluide, le rire comme un refus des catégories figées sont autant de valeurs qui ont été affectées à la notion queer. D’après l'historiographie que l'on propose, il est donc tout à fait compréhensible que l'homosexualité de la fin des années 1970 et, plus sensiblement, dans les années 1980 occupe cet espace politique, avec bien d'autres minorités. Toutefois, les limites identitaires, pour aller vite les tendances essentialistes, justifient l'emploi du terme queer. Le queer marque la résistance à un désir de normalisation, un désir peu propice à la créativité et au questionnement politico-esthétique. Cette résistance a pourtant à voir avec un rire présent dès les années 1970 et 1960, un rire se moquant du genre souvent via la pratique du travestissement, de l'exagération et de la parodie.

\section{Subversion de soi}

\section{Rire queer camp : subversion du genre}

L'utilisation camp, c'est-à-dire un rire subversif, voire absurde, révélant les frontières du genre masculin et féminin, est une innovation importante à la source d'une esthétique recourant fréquemment au travestissement. La notion camp dépolitisée par les célèbres « notes » de Susan Sontag dans les années 1960 a depuis l'ouvrage de Meyer repris des couleurs militantes en revendiquant un positionnement queer (Meyer 1). En 1972, Esther Newton dans son étude anthropologique sur les travestis des années 1960, Mother Camp, avait réaffirmé la nature homosexuelle du camp (Newton 105) et son lien au stand-up pratiqué dans les bars et les cabarets (Newton 56). De la même façon, Laurie Stone noue le camp, le stand-up et le théâtre en incluant dans son ouvrage le Théâtre du Ridicule (Theater of the Ridiculous) des années 1970 et 1980, en particulier Charles Ludlam et ses héritiers dont Charles Busch dans les années 1980 et 1990 avec son Theater in Limbo. Comme le rappelle un critique à propos de ce genre :

Above all, the Ridiculous sought, through laughter (...) a debunking of all mythologies that make this world, in the eyes of the Ridiculous, a place of unremitting insubstantiality. It viewed the world as bereft of all moorings, an ephemeral universe built around illusions and adrift in an ever-expanding black hole of simulacra. (Dasgupta \& Marranca xi)

Cette filiation masculine inspirera un camp lesbien principalement nourri dans l'enceinte du «WOW Café » (Lemoine 2018: 59) à New York, avec la troupe des « Split Britches " (les comédiennes Peggy Shaw et Lois Weaver venant des troupes pratiquant le camp « Hot Peaches » et " Bloolips »), et d'autres artistes tells que Holly Hughes et les cinq comédiennes de la troupe de théâtre « The Five Lesbian Brothers » (Lemoine 2008 : 331). Il est intéressant de relever que ces spectacles ont été beaucoup étudiés non pas en tant que stand-up comique mais plutôt en tant que pratique renouvelant le théâtre lesbien ${ }^{10}$. Leur inclusion dans une tradition comique, comme le suggère l'ouvrage de Stone, s'accorde avec l'idée que l'on a affaire à des formes ouvertes qui interrogent le(s) genre(s) et empruntent à de multiples traditions scéniques. Ces modes de représentation mettent en exergue un rire sans concession sur les contradictions, les 
obsessions et l'éclatante noirceur du désir lesbien ${ }^{11}$. Ce rire camp se moque aussi bien de la culture de masse que de la culture lesbienne, formulant une autocritique queer sur les conséquences de la vie publique LGBT résultant sans doute de la visibilité, de l'engagement et de l'action. Autre contribution majeure du camp lesbien au rire queer est sa déconstruction du genre. Si Butler a pu montrer la dimension performative du genre grâce à la pratique spectaculaire des drag queens, (Butler 1990) le rire camp pourrait bien révéler par l'absurde ridicule que le genre n'est rien (pour faire écho à la formulation de Borch-Jacobsen 34). Le travestissement camp voilerait alors ce rien pour provoquer un rire qui serait le souffle soulevant subrepticement ce voile.

Plus proche du stand-up dans la forme, le spectacle cabaret camp Kiki and Herb est né dans les bars du East Village à la fin des années 1990, puis est devenu pérenne dans divers espaces dont le "Flamingo East» (1998), et finalement a été produit sur Broadway sous le titre : Kiki \& Herb: Alive on Broadway en 2006. Le show alterne récits et chansons, à l'instar des spectacles de Sandra Bernhard ou de Romanovsky \& Phillips (Karvoski 55-63). Malgré l'absence d'une intrigue dramatique, Kiki (Justin Bond, performer) et Herb (Kenny Mellman, pianiste) racontent une histoire fantasque qui théâtralise le rire tout en restant proche de la structure à sketchs du stand-up. Grâce à son personnage Kiki, Bond produit une série de situations grotesques. Par les jeux de mots puisés dans la parodie musicale (« it's better to be knocked down than knocked up " s'inspirant du titre de la chanson "I get knocked down » de "Chumbawamba ", 1997), le performeur transgenre convoque sa condition improbable de femme biologique. Le récit d'une maternité difficile en décalage avec le corps travesti de Bond conduit à rire non pas en guise de lazzis et de quolibets mais d'émerveillement absurde à la fécondité d'un imaginaire enfantant des identités fluides. Cette sensation de fantastique est magnifiée par la ballade musicale composée par les numéros qui tressent la fiction des chansons avec le corps queer incarnés par Kiki, Herb et leur public fidèle ${ }^{12}$. Le camp dopé au glam rock de Bond chanteur révèle l'absurdité du genre binaire engoncé dans des normes étroites comme une célébration extatique d'un moi queer qui partagé dans l'espace public crée l'énergie pour faire communauté et suggérer une stratégie collective de résistance queer.

Cette dimension politique du camp est portée directement par la drag queen Joan Jett Blakk (créée par Terrence Smith à Chicago en 1989 pour les groupes engagés : Queer Nation et Radical Fairies) lorsqu'elle s'est présentée comme la candidate aux élections municipales de Chicago en avril 1991 pour Queer Nation avec des slogans tels que "Putting the Camp into campaign" (cité par Moe 6). Le rire camp fuse lorsqu'elle parodie la reine des talk-shows Oprah Winfrey (sa muse) : «Plus, we [Winfrey \& Blakk] have the same sense of style, except I'm more like Oprah on ecstasy - too much makeup and too much jewelry. » (Karvoski 159). L'excès est donc un style assumé jusqu'aux plus hautes sphères de la représentation: la présidence des États-Unis. L'annonce de sa candidature à la présidentielle américaine contre Bush en 1992 toujours avec le groupe militant Queer Nation affirme l'utilisation du rire camp, à la suite d'ACT UP, à des fins contestataires de l'ordre social dominant. Elle crée une sorte de spirale vertigineuse des référents dans un dédoublement permanent entre des répétitions critiques et ridicules comme cette obsession vestimentaire réelle et fausse à la fois : «I'm wearing my faux faux Channel » s'amuse-t-elle à dire tout en raillant le débat sur les homosexuels dans l'armée : «We don't even need a military, much less gays in it ! If I'm elected president, we'll have Dykes on Bikes protecting this country! (Karvoski 160). Le rire devient 
sérieux, ce qui est un contre-sens pour certains parmi les activistes gays et lesbiens en position de pouvoir (Moe 5-7), tandis que d'autres y voient la combinaison esthéticopolitique queer nécessaire pour perturber les hiérarchies dominantes et proposer une reconfiguration culturelle queer, voire un anarchisme queer. Ce qui est intéressant, c'est que le rire se déplace pour faire apparaître les contradictions, les convulsions entre les communautés queer/non queer et non plus simplement entre des groupes sexuels, raciaux ou de classes. Blakk rit avec la communauté pour ses travers dont elle n'est pas exempte. Elle rit d'elle-même, de ses obsessions et de son plaisir d'être dépassée par l'ambiguïté sexuelle régnant à San Francisco :

Baby, the wimmin here put the boys to shame, you hear me? Foxy, foxy, foxy. (And it's great when you're on the People's Limo [public transportation] watching faces of other passengers - well, a few of them, all right ? - as they try to figure out who's a girl becoming a boy or a boy becoming a girl. I love that!) Girlies rule in San Francisco. (Karvoski 163)

La fluidité des genres devient une source de rire collaboratif et constructif dans l'espace public et non plus un point de terreur et de dissolution du sujet. Cet engagement du rire n'est pas sans évoquer les utopies queer défendues par de nombreux universitaires (Muñoz, Dolan ${ }^{13}$ ) comme source de projections créatives et collectives. On pourrait alors parler d'un rire queer utopique qui se place "sur l'horizon » (Muñoz 2009 : 11).

Cette perspective coexiste avec les pratiques du passé où le travestissement construisait et déconstruisait l'illusion du genre. Le couple plaisir/peur provoqué par l'oscillation entre les deux sexes était mis en exergue et, dans une certaine mesure, l'emprise normative rend cet acte transgressif encore aujourd'hui. Dans le Vaudeville de la fin du dix-neuvième siècle et du début du vingtième siècle (1875-1925), des numéros de travestis tel celui de Raymonde qui à la fin de son spectacle enlève une perruque pour apparaitre comme un homme puis comme une femme puis comme un homme (Allen 578) illustre le frisson comique lié au ressort de la transgression du genre. Ce concentré du coup de théâtre du genre est aussi celui souligné par Esther Newton où certains spectacles travestis des années 1960 se concluent sur la révélation et la fixation du genre par la preuve du sexe masculin révélé par la nudité finale (Newton 44-45) ${ }^{14}$. Au-delà de ces rythmes binaires, le rire camp queer serait alors à tout le moins un brouillage qui témoigne d'une prise de liberté face aux normes sociales à travers une prolifération d'identités sexuelles, raciales etc: butch (ouvrière), femme (chic), folle (faible), travesti.e (brouillage des codes), transgenre (brouillage biologie/ culturel). On retrouve ce rire camp transgenre dans la pièce de Kate Bornstein Hidden : À Gender qui lui permet d'explorer et d'exposer les questions transgenres (Lemoine 2008). Si le rire camp fête la pluralité, il ne s'exonère pas des angoisses existentielles accrochées aux multiples identifications queer. L'exploration des expressions collectives queer s'adonne à un rire qui fouille tous les recoins de l'âme comme avec le camp lesbien. En d'autres termes, de nombreux comiques se moquent d'eux-mêmes bien sûr et de leur communauté.

\section{Rire queer auto-critique : désintégration de l'être?}

42 La subversion des genres et du genre se déploie chez les comiques à travers de nombreuses critiques de la société et de soi-même. Cette autocritique, peut évoquer la tradition de la satire où les travers de l'humanité sont exagérés. Le reflet grossissant 
d'une subculture ajoute peut-être une sorte d'hyper-conscience de la fragilité de l'humain, mais paradoxalement renvoie le marginalisé à son destin général. La comique E.L Greggory prend un malin plaisir à déclarer «I don't like Gay Pride Parades » (Karvoski 187) et à pointer les limites de cette célébration devenue trop festive et commerciale pour certains (promoteurs de la Gay Shame) ou trop instrumentalisée pour ses personnages stéréotypés comme pour Greggory.

Sur un ton léger mais non moins caustique Marga Gomez se moque aussi des images tendues par la société aux lesbiennes, ici à travers la série télévisée The $L$ Word (Showtime, 18 juillet 2004 - 8 mars 2009) sur la vie des lesbiennes de Los Angeles. Gomez dans «Laugh Baby Laugh » joué en avril 2007 à La Pena, Berkeley, Californie raille la représentation affadie des lesbiennes butch dans la série. Cette faiblesse a été soulignée lors d'une rencontre entre les universitaires lesbiennes Jill Dolan et Sue-Ellen Case autour de la conférence d'ATHE (Association for Theatre in Higher Education) à San Francisco en 2004. Les reproches soulignaient une tendance à représenter des lesbiennes aseptisées, vivant dans un milieu très aisé, en évitant des personnages à la féminité masculine moins consensuelle. La caricature de Gomez est donc pertinente et fonctionne car les effets normatifs démasqués déclenchent généralement le rire. Gomez s'en prend aussi au personnage mièvre de Jenny (Mia Kirshner) dont les jérémiades lui donnent envie de faire avance rapide ("fast forward through Jenny»). Outre la remarque qui fait mouche (comme le confirment les rires et aussi les nombreux commentaires à la suite de cet extrait disponible en ligne), l'idée de pouvoir contrôler les éléments extérieurs renforce la portée de la plaisanterie. Le désir et l'impossibilité de cette toute-puissance - faire avance rapide sur tous les mauvais moments - se combinent à la spécificité du déficit de représentation des lesbiennes. Le moment est magnifié entre un trop plein et une absence radicale, lieu où se loge la désidentification défendue par Muñoz dans son ouvrage éponyme. L'introduction de son ouvrage s'ouvre d'ailleurs sur l'analyse d'une scène d'un spectacle de Gomez (Marga Gomez is Pretty, Witty, and Gay, 1992) où la comique transforme la représentation négative d'une lesbienne à la télévision dans une émission des années 1950 en un spectacle érotique (Muñoz 1999: 3, 33). C'est cette expérience de réinvention comique et critique qui anime aussi la puissance du couple butch/femme que Gomez, comme de nombreuses comiques lesbiennes (Holly Hughes, «Split Britches » dans le style de la performance; Lynn Lavner et sa Butch fatale ou Suzanne Westenhoefer, Lea DeLaria etc...), explore amplement. Le couple sulfureux est une source d'humour car il signale à la fois la difficulté d'être représentée et la fabrique d'une nouvelle figure rebelle. Le comique queer permet peut-être alors de dépasser certaines impasses des politiques de la visibilité. La capacité de se moquer des clichés des hétérosexuels mais aussi des homosexuels, même s'il ne s'agit pas d'une équivalence des points de vue, montre la force des stéréotypes qui aident sans doute à délimiter les angoisses et les peurs qui définissent la perception de l'altérité et des limites du sujet. C'est au cœur de ces tensions extrêmes que se trouve l'origine du rire qui masque et dans le même temps manifeste la limite la plus radicale du sujet : sa mort.

Bien sûr, le rire queer a été en première ligne avec les années SIDA. Si l'utilisation de l'humour et du rire est plutôt associée à une seconde génération de pièces sur le SIDA (Jones), certains comiques gays ont pu se saisir de cette crise directement et rapidement. Renouant avec la tradition morbide, Steve Moore (Karvoski 119-123) se moque de sa séropositivité grâce à tous les ressorts transgressifs de l'humour noir. Les 
jeux de mots douteux menant à la mort de l'individu et du sens fusent dans son spectacle. On retrouve là la chute de l'humain dans sa condition de perte. Le style plutôt âpre est accentué par la structure sérielle du stand-up qui enchaîne sans transitions les blagues funestes :

The other day I went to the video store and rented Longtime Companion, And the Band Played On, Philadelphia and An Early Frost. The clerk asked me to pay cash in advance. I think that's mean.

Can you imagine the AIDS ward in heaven?! The good news is, I may get to meet Rock Hudson. The bad news is, I may have to listen to Liberace music for the rest of eternity! (Karvoski 121)

Toutefois dans la version filmée du spectacle Drop Dead Gorgeous (A Tragi-Comedy): The Power of HIV-Positive Thinking (HBO, 1997) un fil narratif autobiographique donne l'impression d'un travail plus classique qui emprunte au style confessionnel mais tout en restant un spectacle comique architecturé par les rires chronométrés. Moore mobilise ici le matériau habituel des comiques: la culture populaire. À la frontière entre grand public et contrepublic, la blague du magasin de location vidéo témoigne d'une culture gay à la fois spécifique, avec la liste des films et téléfilms abordant le SIDA, et générale avec l'évocation de figures célèbres (le pianiste Liberace, la star de cinéma Hudson) dont le SIDA a mis sur la place publique l'orientation sexuelle. La présence du SIDA dans la comédie participe à la fonction du rire qui étend la définition de la sphère publique et de l'humain dans la mesure où la maladie, bien que rejetée par tous, s'impose à la société. Le SIDA, bien sûr, a été l'objet d'une marginalisation forcée entre autres pour les homosexuels masculins, les africains-américains, les immigrés mais l'humour ici vient justement éroder ces restrictions pour rappeler ce qui est au bout de la maladie : la mort. C'est aussi ce qui est au bout du rire : la chute, la mortalité, le rien de l'humain. Et pourtant la sexualité résiste, accroche comme désir de vivre sur la brèche qui soude et sépare l'individu de son environnement. La version filmée du spectacle oppose le rire à la mort aussi dans la mesure où l'artiste est devant les spectateurs qui témoignent de son existence, de sa résistance. Même si le début du numéro sur le SIDA déclenche peu de réactions de la salle, Moore finit par faire rire le public du «Comedy Club» (Los Angeles). Les spectateurs peuvent rire peut-être aussi car finalement grâce au progrès des traitements (expliqué dans le spectacle), l'on croit voir la vie triompher sur la mort, ne serait-ce qu'un instant. Et finalement, n'est-ce pas cela aussi le rire : une victoire, même brève sur la souffrance, l'oppression et la mort?

\section{Conclusion}

Laurie Stone nous rappelle que le rire et le sexe sont intimement liés même si les comiques provoquent l'émotion avec les mots plutôt qu'avec leurs corps :

Like sex, comedy is irrepressible, laughter libidinous, though comedians themselves are often sexually masked, funneling aggression through language rather than their bodies. (Stone xv)

Bien sûr, on comprend la différence entre la dimension physique d'un acte sexuel et la corporalité d'un.e comédien.ne, néanmoins l'utilisation du corps reste un ingrédient fondamental dans le genre du stand-up. L'énergie, la présence, les mimiques, la plasticité du visage, les ondulations des membres sont autant d'expressions du corps qui sculptent les mots, habitent l'espace et le temps du plateau. L'on peut voir d'ailleurs tout un éventail de stratégies pour faire exister le corps queer dans l'espace scénique : 
allant de l'énergie débordante de DeLaria au calme olympien de Tigo Notaro en passant par le camp flamboyant de Joan Jett Blakk ou Justin Bond. Tous ces artistes ont su à la fois ouvrir des espaces pour un comique dont le fond reste le rire mais dont la forme a su questionner, voire déstabiliser les limites normatives qui tentent sans cesse de policer l'individu et son environnement.

Le stand-up queer s'inscrit donc pleinement dans l'histoire de la performance queer même s'il ne jouit pas de la même aura. Selon les propos de Zoglin, la part des comiques dans la transformation de la culture américaine a trop souvent été négligée :

The stand-up comics of the late " 60 s and ' 70 s are the forgotten stars of this cultural revolution. At a time when young people were challenging the power structure and freeing their minds, stand-up comics struck an especially responsive chord. (Zoglin 4)

Si la périodicité diffère, le stand-up queer pourrait aussi avoir été délaissé par la critique queer. Peut-être parce qu'il existe une porosité des genres où le stand-up se transforme en performance solo (Tim Miller, Holly Hughes etc.), en performance drag queen (Kiki and Herb, Busch etc.) ou drag king (Peggy Shaw, Dred, etc.) et parfois le stand-up devient une satire sociale (Moore, « Pomo Afro Homos »). La circulation du rire queer permet de desserrer un peu plus l'étau oppressif, tout particulièrement en travaillant dans la transversalité, l'intersectionnel, la désidentification comme les hommes noirs gays de «Pomo Afro Homos» ou les comiques latinas lesbiennes (Maria Gomez, Carmelita Tropicana) et en créant un contrepublic. Le stand-up contribue à donner une forme, une esthétique à des mouvements politiques, mais une politique qui passe par la prolifération et l'hybridation de formes culturelles évanescentes. Comme le suggère l'action camp queer de Joan Jett Blakk, le mélange d'une élection politique et d'un style camp fait rire ou trembler non parce qu'il oppose un groupe sexuel à un autre mais parce que cette hybridation questionne la société toute entière. Le rire queer est donc à la poursuite d'une réouverture, même éphémère, des possibles tant sur le plan individuel que social. Au fur et à mesure de l'intégration gay et lesbienne, le risque de l'évaporation d'un rire homo risque d'être total. Toutefois, le positionnement queer tente de déjouer les rabattements normatifs pour réintroduire le rire queer sur l'horizon et maintenir le décalage, l'asymétrie qui est à la source de l'éclat de rire libérateur, provocateur et révolutionnaire.

--- « Hedwig and the Angry Inch, dialectiques queer du spectacle drag ». GRAAT On-Line 19 (July 2017) : 40-65.

--- « Performing Black Queerness in the U.S. South: Ethnographic Performance and the Blurring of Presence in E. P. Johnson's Sweet Tea». Études anglaises 69/2 (Avril-Juin 2016) : 176-193.

--- « Métamorphose du genre : sang et technologie ». La Fabrique du genre. Ed. Sophie Marret et Claude Le Fustec. Rennes : Presse Universitaire de Rennes. 2008, 45-63.

«Le Camp lesbien des Five Lesbian Brothers ». Etudes Anglaises 61/3 (juillet-septembre 2008) : 330-338.

Limon, John. Stand-up Comedy in Theory, Or, Abjection in America. Durham : Duke University Press, 2000.

Marche, Guillaume. La militance LGBT aux États-Unis : sexualité et subjectivité. Lyon : Presse Universitaire de Lyon, 2017. 
Mizejewski, Linda. Pretty/Funny: Women Comedians and Body Politics. Austin : University of Texas Press, 2014.

Meyer, Moe, ed. The Politics and Poetics of Camp. Londres et New York : Routledge, 1994.

Muñoz, José Esteban. Cruising Utopia: The Then and There of Queer Futurity. New York : New York University Press, 2009.

--- Disidentifications: Queers of Color and the Performance of Politics. Minneapolis : University of Minnesota Press, 1999.

--- Moore, Steve. Drop Dead Gorgeous (A Tragi-Comedy): The Power of HIV-Positive Thinking. HBO, 1997. 5 octobre 2017. < https://www.youtube.com/watch?v=PvD75kWxikw >

Newton, Esther. Mother Camp: Female Impersonators in America. Chicago: The University of Chicago Press, 1972/1979.

Nancy, Jean-Luc. « Le Rire, la présence ». Critique « Quatre essais sur le rire » 488-489 (JanvierFévrier 1988) : 41-60.

Panisch, Alex. « Just for laughs, here are 12 queer comics you should know. » Out. Tue, 2015. 15 septembre 2017. < https://www.out.com/entertainment/popnography/2014/04/02/newlegendary-12-queer-comics-you-should-know >

Plum, Jay. «Pleasure, Politics and the Performance of Community: Pomo Afro Homo's Dark Fruit ». Modern Drama 34:1 (Spring 1996) : 117-131.

Riggs, Marlon. « Black Macho Revisited: Reflections of a Snap! Queen». Black American Literature Forum 25:2 (Summer 1991) : 389-394.

Román, David. Acts of Intervention: Performance, Gay Culture, and AIDS. Bloomington \& Indianapolis : Indiana University Press, 1998.

Russell, Mark, ed. Out Of Character: Rants, Raves, and Monologues from Today's Top Performance Artists. New York : Bantam Books. 1997.

Smith, Terrence. « Joan Jett Blakk Announces Candidacy. » 1992. 25 septembre 2017.

< http://mediaburn.org/video/joan-jett-blakk-announces-candidacy >

Sykes, Wanda. «I'ma Be Me ». HBO TV Special. 10 Octobre 2009. March 1st, 2018

< https://www.youtube.com/watch?v=1_wWJ-_4uSY >

Warner, Michael. «Publics and Counterpublics». Quarterly Journal of Speech, 88:4 (November 2002): 413-425.

Weber, Samuel. « Le temps d'un rire ». Traduction Hélène Vilavella-Johnson et Mikkel BorchJacobsen. Critique "Quatre essais sur le rire" 488-489 (Janvier-Février 1988) : 61-76.

Wilmeth, Don B \& Miller Tice L. Eds. Cambridge Guide to American Theatre. Cambridge: Cambridge University Press, 1996.

Zoglin, Richard. How Stand-up in the 1970s Changed America. New York, London, New Delhi:

Bloomsbury, 2008. 


\section{BIBLIOGRAPHIE}

Allen, Fred. «The Life and Death of Vaudeville ». 1956. In The American Stage. Ed. Laurence Senelick. New York : The Library of America, 2010. 567-585.

Borch-Jacobsen, Mikkel. « Bataille et le rire de l'être ». Critique "Quatre essais sur le rire" 488-489 (Janvier-Février 1988) : 16-40.

Bornstein, Kate. Hidden: A Gender. In Gender Outlaw: On Men, Women, and the Rest of Us, Kate Bornstein. New York : Vintage Books. 1995, 169-223.

Butler, Judith. Bodies That Matter: On the Discursive Limits of "Sex". New York : Routledge, 1993.

--- Gender Trouble: Feminism and the Subversion of Identity. New York : Routledge, 1990.

Case, Sue-Ellen. Split Britches: Lesbian Practice/Feminist Performance. New York : Routledge, 1996.

Conquergood, Dwight. « Performing as a Moral Act: Ethical Dimensions of the Ethnography of Performance». Literature in Performance 5.1 (1985) : 1-13.

Crenshaw, Kimberlé. « Mapping the Margins: Intersectionality, Identity Politics, and Violence against Women of Color». Stanford Law Review 43:6 (1991) : 1241-1299.

Daring C.B, Rogue J, Shannon Deric, Volcano Abby. Eds. Queering Anarchism: Addressing and Undressing Power and Desire. Oakland, Edinburgh, Baltimore : AK Press, 2012.

Dasgupta, Gautam. «Preface to the 1998 Edition. » In Theatre of the Ridiculous. Ed. Bonnie Marranca et Gautam Dasgupta. Baltimore, Maryland : PAJ Books. 1998, vii-xi.

Davy, Kate. Lady Dicks and Lesbian Brothers: Staging the Unimaginable at the WOW Café Theatre. Ann Arbor : University of Michigan Press, 2011.

Dolan, Jill. « Utopia and the 'Utopian Peformative' ». Theatre Journal 53:3 (October 2001) : 455-479.

--- Presence and Desire: Essays on Gender, Sexuality and Performance. Ann Arbor : University of Michigan Press, 1993.

--- The Feminist Spectator as a Critic. Ann Arbor : University of Michigan Press, 1988.

Gilbert, Joanne R.

--- « Chapter 15: Lesbian Stand-Up Comics and the Politics of Laughter. » In Women and Comedy: History, Theory, Practice. Ed. Peter Dickinson, et.al. Maryland : Farleigh Dickinson University Press. 2013, 184-196.

--- Performing Marginality Humor, Gender, and Cultural Critique. Detroit, Michigan : Wayne State University Press, 2004.

--- « Performing Marginality: Comedy, Identity, and Cultural Critique. » Text and Performance Quarterly 17 (1997) : 317-330.

Edgecomb, Sean F. Charles. Ludlam Lives !: Charles Busch, Bradford Louryk, Taylor Mac and the Queer Legacy of the Ridiculous Theatrical Company. Ann Arbor: University of Michigan Press, 2017.

Gomez, Marga. «Bar Tab. » Bay Area Reporter. March 2012. 5 octobre 2017

< http://www.epochalips.com/2012/04/gay-comedy-beginnings-in-sf/ > 
--- « Laugh Baby Laugh » recorded in April 2007 at La Pena in Berkeley, CA. 4 octobre 2017.

< https://www.youtube.com/watch?v=DZtJ76Apv3A. >

Hutcheon, Linda. A Theory of Parody: The Teachings of Twentieth-Century Art Forms. Urbana \& Chicago: University of Illinois Press, 1985/2000.

Jones, Therese. Ed. Sharing The Delirium: Second Generation AIDS Plays and Performances. Portsmouth, NH: Heinemann, 1994.

Johnson, Patrick E. Appropriating Blackness: Performance and the Politics of Authenticity. Durham, N.C. : Duke University Press, 2003.

Kivy, Peter. « Jokes Are a Laughing Matter. » The Journal of Aesthetics and Art Criticism 61:1

(February 2003) : 5-15.

Lemoine, Xavier. « Performer l'intersectionnalité à la fin du XXe siècle : Carmelita Tropicana, lesbienne cubaine au cœur du Village new-yorkais », Horizon/Théâtre 10-11 (2018) : 58-76.

\section{NOTES}

1. Dans son étude Esther Newton relève l'importance de deux types de performance comique : le «slapstick» principalement visuel et le stand-up principalement verbal (Newton 52). Cette dernière est considérée comme centrale dans la culture homosexuelle et elle est associée au camp : "The stand-up comic is the specialized, professionally performing version of the bestdefined role figure in homosexual life, the campy queen. » (Newton 56)

2. Voir les réflexions des universitaires queer Michael Warner (Warner 423-424) et José Muñoz (Muñoz 1999: 147-149).

3. L'utilisation des termes gays et lesbiens, LGBT, queer sont assez flottants selon les acteurs concernés. Il existe une prédominance des termes gays et lesbiens dans les années 1980 tandis que « queer » va venir s'opposer aux tendances essentialistes et normatives du mouvement gay et lesbien. LGBT s'impose aussi au cours des années 1980 de façon plus consensuelle. Ici tout en gardant ces grandes tendances en tête, on respecte également la façon dont les acteurs envisagent les choses quelle que soit la période.

4. Samuel Weber résume la position de Freud: «Le mot d'esprit n'est un mot d'esprit que par l'effet du rire qu'il provoque mais qui en retour le constitue pour ainsi dire rétrospectivement " (Weber 64)

5. Cette idée de la réappropriation de l'humour d'autodénigrement (« self-deprecating humor ») est repérée par Gilbert (Gilbert 319) même si bien sûr c'est une forme utilisée par de nombreux comiques (mais avec un sens différent selon la position des groupes représentés). Cet espace de liberté fait aussi écho au processus de désidentification.

6. Le "zap» est une interruption d'un événement souvent public dans le but d'exposer un problème ici l'homophobie. Cette pratique utilisée par le Gay Liberation Front dans les années 1970 sera ensuite un type d'intervention militante fréquemment utilisée par ACT UP.

7. Comme le rappelle Lynda Montgomery: «People are always interested when you reveal something personal about yourself » (Karvoski 202)

8. Voir l'article fondateur de Crenshaw qui veut mettre fin à une analyse sectorielle et disciplinaire afin de ne pas répéter les exclusions subies par les diverses minorités et dans le but de confronter simultanément les oppressions multiples.

9. « (...) disidentification is the hermeneutical performance of decoding mass, high, or any other cultural field from the perspective of a minority subject who is disempowered in such a representational hierarchy » (Muñoz 1999: 25) 
10. On pense ici aux auteures Jill Dolan, Kate Davy, Sue-Ellen Case pour citer les plus connues qui ont, elles-mêmes, renouvelé le critique théâtrale féministe et lesbienne.

11. Voir par exemple Dress Suits To Hire de Holly Hughes jouée à sa création par Weaver et Shaw.

12. Dans le public des spectacles auquel j'ai assisté en 1997-1998, se retrouvait le contrepublic d'étudiants inscrits aux divers séminaires queer des universités newyorkaises et aussi de leurs professeurs dont Muñoz, Michael Moon, etc.

13. Dolan conclut son article sur la proposition suivante: "This, for me, is the beginning (and perhaps the substance) of the utopic performative: in the performer's grace, in the audience's generosity, in the lucid power of intersubjective understanding, however fleeting." (Dolan 479)

14. Newton souligne que ce final est plus courant dans les lieux « hétérosexuels ». Toutefois, on retrouve une fin similaire à la fin des années 1990s dans la comédie musicale queer, Hedwig and the Angry Inch. (Lemoine 2017)

\section{RÉSUMÉS}

Dans le monde du spectacle, les comiques ont souvent été relégués au rang d'art mineur, voire rejetés comme un pur produit commercial. Et pourtant la tradition du stand-up, construit sur l'enchaînement rapide de blagues, ne cherche-t-elle pas à saisir l'essence même du rire? L'histoire des comiques LGBTQ (lesbien, gay, bisexuel, transgenre et queer) a été également négligée dans l'histoire du théâtre et de la performance queer. Cet article revisite donc ces histoires pour essayer d'identifier le rôle d'un rire queer à travers ses fonctions et ses effets théoriques. En explorant les dimensions politiques et esthétiques des comiques gays, lesbiens et transgenres depuis les années 1960, on peut comprendre le rôle du rire dans la constitution d'une pratique dissidente face aux normes et à la domination hétéronormatives blanches. Cette résistance du rire est articulée aux notions théoriques queer de désidentification et de contrepublic qui traversent les trois voies empruntées ici : le rire politique, performatif et subversif.

In the world of performance art, comedians have often been considered as minor artists or even have been rejected as mere commercial products. Yet doesn't the stand-up tradition - defined by the delivery of fast paced jokes - seek to grasp the essence of laughter? The history of LGBTQ comedians has equally been neglected by the queer theater and performance history. This paper aims at revisiting these histories in order to account for the role of queer laughter thanks to its theoretical and functional effects. By exploring the political and esthetic dimensions of gay, lesbian and transgender people since the 1960s, we can understand the role of laughter in the construction of dissenting practices against white heteronormative norms and domination. This resistance through laughter is articulated here through the queer theoretical notions of dissidentification and counterpublicity present in the three paths taken by this work: political, performative, and subversive laughter. 
INDEX

Keywords : queer, comedians, stand-up, gay, lesbian, transgender, race, dissidentification, counterpublic, performance, camp, homophobia

Mots-clés : queer, comiques, stand-up, gay, lesbien, transgenre, race, désidentification, contrepublic, performance, camp, homophobie

\section{AUTEURS}

\section{XAVIER LEMOINE}

Maître de conférences

Université Paris-Est Marne-la-Vallée, Laboratoire LISAA (LIttératures SAvoirs et Arts)

xavier.lemoine@u-pem.fr 